\title{
Journal of Comprehensive Pediatrics
}

\section{The Importance of Vaccination Program Overview}

\author{
Mohammad Reza Boloursaz ${ }^{*}$ \\ ${ }^{1}$ Pediatric Respiratory Disease Research Center, NRITLD, Masih Daneshvari Hospital, Shahid Beheshti University of Medical Sciences, Tehran, Iran \\ * Corresponding author: Mohammad Reza Boloursaz, Pediatric Respiratory Disease Research Center, NRITLD, Masih Daneshvari Hospital, Shahid Beheshti \\ University of Medical Sciences, Tehran, Iran.Tel.:+98-2127122458, Fax: +98-2126109484, E-mail: bolursazm@yahoo.com
}

Keywords: Vaccination; Rotavirus vaccine; Pneumococcal vaccine

Vaccines are the most effective tools for preventing of infection-associated mortality and morbidity. Vaccination as one of the most successful public health achievements of the 20th century is one of the best available weapons to protect children against dangerous childhood diseases. In fact due to the hygiene observance, better nourishment, prevention of crowding in a confined space, antibiotics and above of all now available vaccines, many diseases which were life threatening in past, are preventable and rarely seen today.

It used to be the world wide norm that a large number of kids died before adulthood during an infectious disease outbreak; however, by means of immunization such diseases are only in the history today. Several types of vaccines, especially for children, are currently available. These vaccines act effectively and most of them are able to prevent diseases with $90-99 \%$ of effectiveness, thus they are considered as the most important weapon for children's safety.

The large-scale introduction of a new vaccine can uncover many secrets and surprises about the epidemiology of disease that might not be discovered in any other way. Examination of the outcome of a vaccine introduction can validate prior assumptions concerning the burden of disease and the economic consequences of the vaccination program, as well as determine herd effects of the program arising from either a reduction in the environmental load of the infectious agent or a decrease in the susceptible group that might blunt transmission of the agent.

Vaccines which are not currently available in Iran and is hoped that the authorities do a meritorious service with addition of these ones to public health system, contain:

Rotavirus vaccine: Rotavirus is the single most important cause of severe acute gastroenteritis worldwide in children under the age of five. The World Health Organization (WHO) estimates that rotavirus is associated with approximately 527,000 deaths globally, the majority of which $(>85 \%)$ occur in young children in the developing countries of Asia and Africa $(1,2)$. Recently, WHO made a global recommendation for rotavirus immunization in all infants(3)based on the efficacy data generated in developing countries with both commercial rotavirus vaccines namely, Rotarix ${ }^{\mathrm{TM}}$ (GlaxoSmithKline Biologicals) and Rotateq ${ }^{\mathrm{TM}}$ (Merck and Co., Inc) (4).

Haemophilus Influenzae b vaccine: It can protect children against meningitis and throat infection that can cause epiglottitis. Before the advent of this vaccine Haemophilus Influenzae was one of the most important causes of mental retardation in US. This disease is cause

Article type: Editorial; Received: 27 Oct 2012, Revised: 29 Oct 2012, Accepted: 02 Nov 2012; DOI: 10.17795/compreped-8892

-Implication for health policy/practice/research/medical education:

We believe that by modifying the national vaccination program of Iran we can achieve excellent pediatric health status among Middle East countries.

-Please cite this paper as:

Boloursaz MR, The Importance of Vaccination Program Overview. J Compr Ped. 2013:4(1):45-6.

Copyright (c) 2013, Iranian Society of Pediatrics.

This is an Open Access article distributed under the terms of the Creative Commons Attribution License (http://creativecommons.org/licenses/by/3.0), which permits unrestricted use, distribution, and reproduction in any medium, provided the original work is properly cited. 
of the incidence of meningitis in children younger than five years old; especially in six to twelve month old children it causes irreversible brain damage, whereas the vaccine is safe.

Chickenpox vaccine: Varicella is the primary disease caused by the varicella-zoster virus. It is a common and highly contagious disease and has a significant health impact on children. Although the clinical course of varicella is usually mild and self-limiting, varicella does cause complications and mortality resulting in financial expense. Chickenpox or varicella now has been considered one of the most common vaccine-preventable diseases in many countries.This vaccine that has been available since 1995, not only immunizes the person against chickenpox, but also protects her/him against shingles for the whole life.

Meningococcal vaccine: Neisseria meningitidis is a leading cause of bacterial meningitis and septicemia in children and adolescents, with high case fatality and morbidity despite good medical care. Disease rates are highest among children, and slightly elevated rates have been observed among adolescents and young adults. Vaccines against meningococcal disease, based on the polysaccharide capsule, have been in use all over the world. The formulation currently available covers serogroups A, C, Y, and W-135, which have caused approximately two thirds of cases of meningococcal disease in recent years. The currently licensed polysaccharide vaccine is safe and effective, so it is recommended for routine use because of its relative effectiveness in children and its economic major effects on children health system. Widespread use of Haemophilus influenzae type b (Hib) and Streptococcus pneumoniae conjugate vaccines, in which a carrier protein is conjugated to the polysaccharide to produce a T-cell-dependent response, has resulted in dramatic reductions in the burden of disease caused by these two pathogens. A quadrivalent meningococcal conjugate polysaccharide $\mathrm{A} / \mathrm{C} / \mathrm{Y} / \mathrm{W}-135$ vaccine $(\mathrm{MCV}-4)$ is safe and immunogenic in healthy adults. In January, 2005, the US Food and Drug Administration approved MCV-4 for use in adolescents and adults aged 11 to 55 years.

Pneumococcal vaccine: This vaccine protects children against meningitis, pneumonia, sepsis and a specific type of ear infection. Pneumococcal infection is one of the most common causes of death in children that can be prevented by means of the vaccine.

Based on the undeniable effects of these vaccines on children health promotion and preventing the adverse socioeconomic consequences of these diseases we believe that by modifying the national vaccination program of Iran we can achieve excellent pediatric health status among Middle East countries.

\section{Authors' Contribution}

None declared.

\section{Financial Disclosure}

None declared.

\section{References}

1. World Health Organization. Rotavirus vaccines. Wkly Epidemiol Rec. 2007;82(32):285-95.

2. Parashar UD, Burton A, Lanata C, Boschi-Pinto C, Shibuya K, Steele D, et al. Global mortality associated with rotavirus disease among children in $2004 \mathrm{~J}$ Infect Dis. 2009; p. S9-S15

3. World Health Organization. Human infection with new influenza A (H1N1) virus: Mexico, update, March-May 2009. Wkly Epidemiol Rec. 2009;84(23):213-19.

4. Zaman K, Dang DA, Victor JC, Shin S, Yunus M, Dallas MJ, et al. Efficacy of pentavalent rotavirus vaccine against severe rotavirus gastroenteritis in infants in developing countries in Asia: a randomised, double-blind, placebo-controlled trial. Lancet. 2010;376(9741):615-23. 